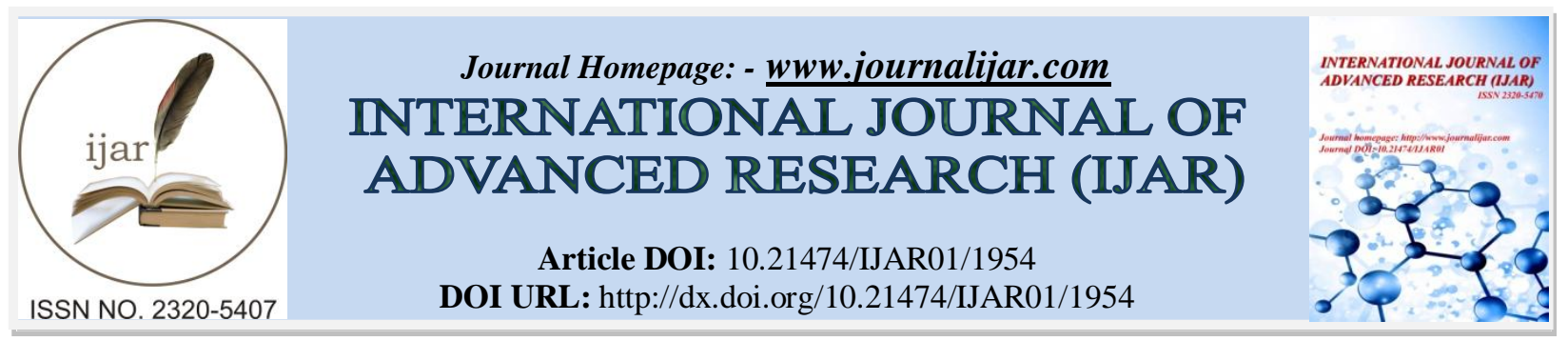

RESEARCH ARTICLE

\title{
THE ELECTRONIC PROPERTIES SIMULATION OF INDIUM NITRIDE (InN) WURTZITE NANOCRYSTAL USING DENSITY FUNCTIONAL THEORY WITH GENERALIZED GRADIENT APPROXIMATION
}

Askander Khalid Kaka and Akram Hashim Taha.

Department of Physics, Faculty of Science and Health, Koya University, Erbil-Iraq.

\section{Manuscript Info}

Manuscript History

Received: 11 August 2016

Final Accepted: 12 September 2016

Published: October 2016

Key words:-

Density functional theory, Large unit

cell, InN wurtzite nanocrystal.

\section{Abstract}

We have stimulated the electronic properties of wurtzite Indium Nitride by using density functional theory (DFT). The energy gap is size dependent at which $E_{\mathrm{g}}$ increase with decreasing the size of $\mathrm{InN}$ nanocrystals. Also the lattice constant behaves in the same way as energy gap do. The cohesive energy is also calculated for the $\mathrm{InN}$ nanocrystals for 4, 8, 32 and 64 core atoms. Results shows that the cohesive energy values become larger as the size of core atoms increases and we predict that when we go for larger sizes the value of the cohesive energy will tend to be the same value of bulk InN.

Copy Right, IJAR, 2016,. All rights reserved.

\section{Introduction:-}

One of the most important classes of the III-nitrides group semiconductor materials are $\mathrm{InN}$, AlN and GaN with their alloys, where they are used for optoelectronic applications such as blue light emitting diodes (LED's) and lasers [1]. Indium Nitride ( $\mathrm{InN})$ is the least explored nitride, due to difficulties in synthesizing high quality single crystal. Very recently, these problems have been overcome and some of the key band parameters have been conclusively determined $[2,3]$. The progress in the fabricating of high performance mobility high electron mobility transistors and light-emitting diodes [4]. Recent successes in the growth of high quality wurtzite InN [5-7], have resulted in substantial interest in the use of $\mathrm{InN}$ in the fabrication of high performance high electron mobility transistors and light-emitting diodes [8]. These types of binary and ternary semiconductor nanocrystals have been studied extensively by many academic workers [9-11]. Confinement of electrons in these nanostructures gives rise to quantum effects, so recent researches concentrated on nanostructures in one dimension (1D), two dimensions 2D and three dimensions 3D leading to quantum well, quantum wires and quantum dot respectively. Many codes have been established to predict the behavior of nanostructures and so many adding to the main programs have been done to be suitable and applicable to find the properties of them. In this work we focused on some of the important properties of indium nitride $(\mathrm{InN})$ semiconductor using the density functional theory (DFT). Periodic boundary condition (PBC) [12] was considered to choose the nanocrystal structure of InN. We also selected the generalized gradient approximation (GGA) for the exchange functional in conjunction with accurate expressions for the correlation functional have led to numerous applications in which density-functional theory (DFT) provides structures, bond energies, and reaction activation energies in excellent agreement with the most accurate ab-initio calculations and with the experiment.

Corresponding Author:- Askander Khalid Kaka.

Address:- Department of Physics, Faculty of Science and Health, Koya University, Erbil-Iraq. 


\section{Theoretical background:-}

The large unit cell method is a kind of supercell method that was suggested and first applied for the investigation of the electronic band structure of semiconductors [13]. The LUC alters the size and the shape of the unit cell so that the symmetry points in the original Brillouin zone at a wave vector $\mathbf{k}$ become equivalent to the central symmetry point in the new reduced zone. The number of atoms in the central cell $(\mathbf{k}=0)$ is increased to match the real number of nanocrystal atoms [14]. LUC coupled with DFT (LUC-DFT) saves the computational time to calculate the electronic properties of the nanocrystal. density functional theory is a ground-state electronic energy which is determined completely by the electron density $\rho(\mathbf{r})$ [15]. In 1927, Thomas-Fermi put the concept of DFT based on the uniform electron gas [16]. They proposed the following functional for the kinetic energy:

$$
T[\rho]=\frac{3}{10}\left(2 \pi^{2}\right) \int \rho^{5 / 3}(\mathbf{r}) d \mathbf{r}
$$

The basic variable in DFT is the single-particle density $\rho(\mathbf{r})$, where for an $N$-particles system $[17,18]$,

$$
\rho\left(\mathbf{r}_{1}\right)=N \int . \cdot \int\left|\psi\left(\mathbf{r}_{1}, \mathbf{r}_{2}, \ldots, \mathbf{r}_{N}\right)\right|^{2} d^{3} r_{2} d^{3} r_{3} \cdot d^{3} r_{N}
$$

DFT formulated by P. Hohenberg and W. Kohn in 1964 [19] with the central idea to replace the many-body problem with an equation for the electron density. Hohenberg and Kohn stated and proved that;

$1^{\text {st }}$ Lemma: The density of ground state $\rho(\mathbf{r})$, uniquely determines the external potential $V(\mathbf{r})$, within an additive constant. The total energy can be found as:

$$
\begin{aligned}
E[\rho] & =T_{e}[\rho]+V_{e-e}[\rho]+V_{e-n}[\rho] \\
& =F_{\mathrm{HK}}[\rho]+\int \rho(\mathbf{r}) V(\mathbf{r}) d^{3} r
\end{aligned}
$$

$F_{\mathrm{HK}}[\rho]$ is a universal functional of $\rho(\mathbf{r})$, which is represent the kinetic energy of the electrons $T[\rho]$ and coulomb interactions energy between electrons-electrons $V_{e-e}[\rho] . V_{e-n}[\rho]$ is coulomb interactions energy between electronsnuclei.

$2^{\text {nd }}$ Lemma: The ground-state energy can be obtained variationally; the density that minimizes the total energy is the exact ground state density $\rho_{\mathrm{o}}(\mathbf{r})$. That is, for a trial electron density $\rho_{t}(\mathbf{r})$;

$$
E\left[\rho_{t}\right] \geq E\left[\rho_{\mathrm{O}}\right]
$$

W. Kohn and L. J. Sham [20] developed the Hohenberg-Kohn theorems to calculate the ground-state energy by consider a non-interacting particles system that generate the same density as any given system of interacting particles [21]. Thus, a general Kohn-Sham energy $E_{\mathrm{KS}}[\rho]$ formulation of DFT is [22],

$$
E_{\mathrm{KS}}[\rho]=T_{s}[\rho]+J[\rho]+V_{e-n}[\rho]+E_{x c}[\rho]
$$

where, $T_{s}[\rho]$ is the kinetic energy of the non-interacting system, $J[\rho]$ is the classical coulomb repulsion energy, and $E_{x c}[\rho]$ is the exchange-correlation energy. The most commonly used of this category is the exchange-correlation energy functional of Perdew, Burke and Ernzerhof (PBE) [23], which is called the generalized gradient approximation (GGA). GGA is usually written as an integral over an exchange-correlation energy density,

$E_{x c}^{\mathrm{GGA}}\left[\rho_{\uparrow}, \rho_{\downarrow}\right]=\int \rho(\mathbf{r}) \in_{x c}^{\mathrm{GGA}}\left[\rho_{\uparrow}, \rho_{\downarrow}, \nabla \rho_{\uparrow}, \nabla \rho_{\downarrow}\right] d^{3} r$

where $\epsilon_{x c}^{\mathrm{GGA}}$ is the exchange-correlation energy per electron of an electron gas with homogeneous spin densities $\rho_{\uparrow}(\mathbf{r})$ and $\rho_{\downarrow}(\mathbf{r})$ in addition to the density gradients $\nabla \rho_{\uparrow}(\mathbf{r})$ and $\nabla \rho_{\downarrow}(\mathbf{r})[24]$. All above equations are evaluated by using Gaussian 03w code [25]. 


\section{Results and Discussion:-}

The InN wurtzite (Fig. 1a) nanocrystal has been studied using the well-known density functional theory coupled with the large unit cell (Figs. 1b-1e) formalism (DFT-LUC).

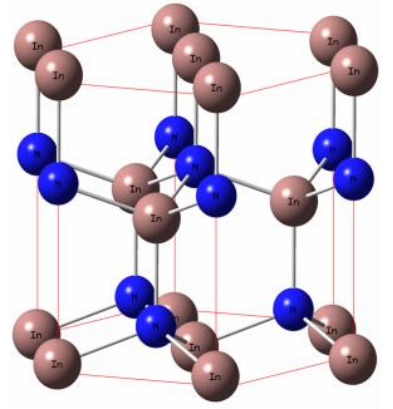

(a)

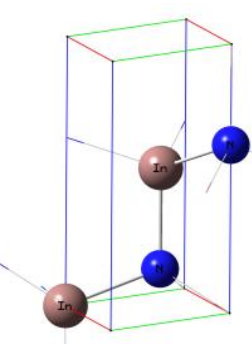

(b)

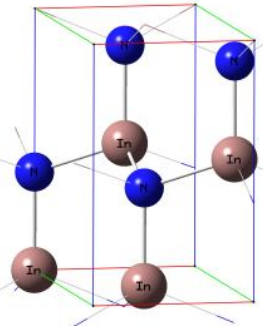

(c)

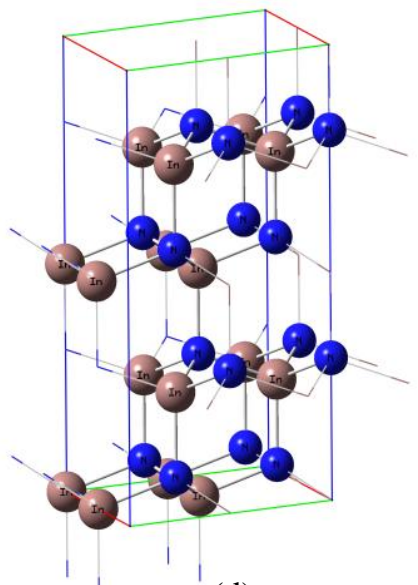

(d)

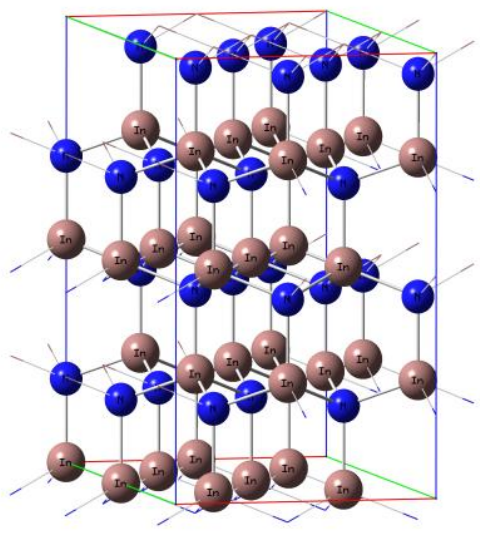

(e)

Figure 1:- a) the wurtzite InN structure, and some of LUCs; b) 4 atoms, c) 8 atoms, d) 32 atoms, and e) 64 atoms per LUC.

The DFT is applicable for the ground state of the system, so we have to find the minimum energy which can be considered as the equilibrium point at a distant called the optimized lattice constant. The equilibrium position is to be found for all core atoms 4, 8, 32 and 64 that is the distant at which both the attractive and the repulsive forces are equal. Fig.(2) shows the minimum energy for 32 core atoms. The optimized lattice constant was found around $(0.341 \mathrm{~nm})$. The same procedure done for 4,8 and 64 core atom with correspondence optimized lattice constants $(0.371,0.347$ and $0.340 \mathrm{~nm})$ respectively. The lattice constant's behavior with the number of core atoms is illustrated in Fig. (3).

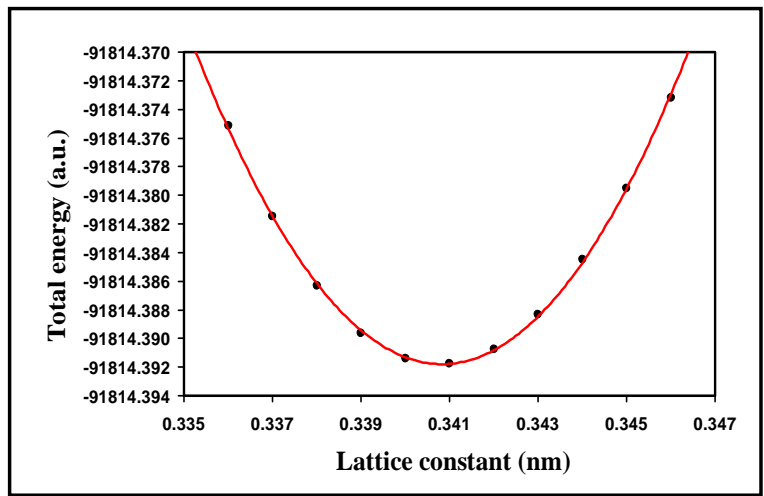

Figure 2:- the optimized lattice constant of InN nanocrystal for 32 core atoms.

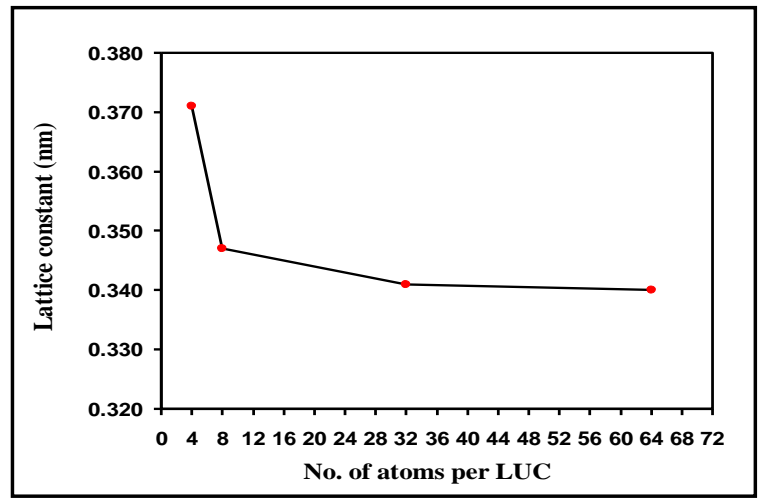

Figure 3:- the relationship between the lattice constant and the core of atoms of InN. 
One of the most important parameter that is a main characteristic for materials is the energy gap. The energy gap is mainly controlled by the nanoparticle's shape, size and composition [26-28]. The energy gap is decreasing as the size of the nanocrystal become large. This result is consistent with the experimental and theoretical results [29].

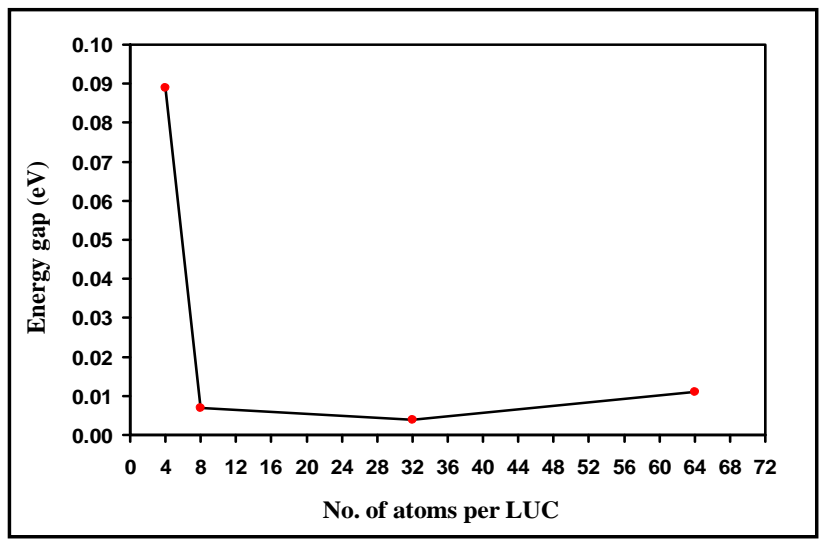

Figure 4:- energy gap of InN wurtzite nanocrystal as a function of the number of atoms per LUC.

Theoretical calculations show that the intrinsic bandgap of $\mathrm{InN}$ is $\approx 0.65 \mathrm{eV}$ [30], while $\mathrm{Wu}$ et al. [31] have pointed out that the intrinsic bandgap wurtzite-structured InN grown on sapphire substrates by molecular-beam epitaxy to be $0.7-0.8 \mathrm{eV}$ which is much lower than the commonly accepted value of $1.9 \mathrm{eV}$. We have reported here the energy gap lies in the infrared region (Fig. 4) while the experimental result by K. Sardar et al. found that the energy gap lies near the infrared region and was around $0.7 \mathrm{eV}$ using a new chemical route [32].

The cohesive energy was found using the following formula[33, 34]:

$$
E_{c o h}^{\mathrm{In}_{n} \mathrm{~N}_{m}}=\frac{E_{\text {total }}^{\mathrm{In}_{n} \mathrm{~N}_{m}}}{(n+m)}-\left(n E_{\text {free }}^{\mathrm{In}}+m E_{\text {free }}^{\mathrm{N}}\right)-E_{\mathrm{o}}^{\mathrm{In}_{n} \mathrm{~N}_{m}}
$$

where $n$ and $m$ are the number of atoms due to In and $\mathrm{N}$ in the core, respectively. $E_{\text {free }}$ is the free atom energy, and $E_{\mathrm{o}}$ represents the correction to the cohesive energy for thevibration energy at ground-state (zero-point). Fig. (5) shows that the cohesive energy for InN nanocrystal for 4, 8, 32 and 64 core atoms, the values $E_{c o h}$ lies between 4.834 and $8.439 \mathrm{eV}$ per atom. The theoritical value found by M. Fuchs et al. using full potential linear augment plane wave (FP-LAPW) is $7.35 \mathrm{eV}$ [35] while the experimental value was $7.172 \mathrm{eV}$ [36]. The values we got is very close to the experimental and theoritical values in Ref. [37]. It is clear that the value of $E_{\text {coh }}$ for InN nanocrystals tends to be almost same value for bulk $\mathrm{InN}$.

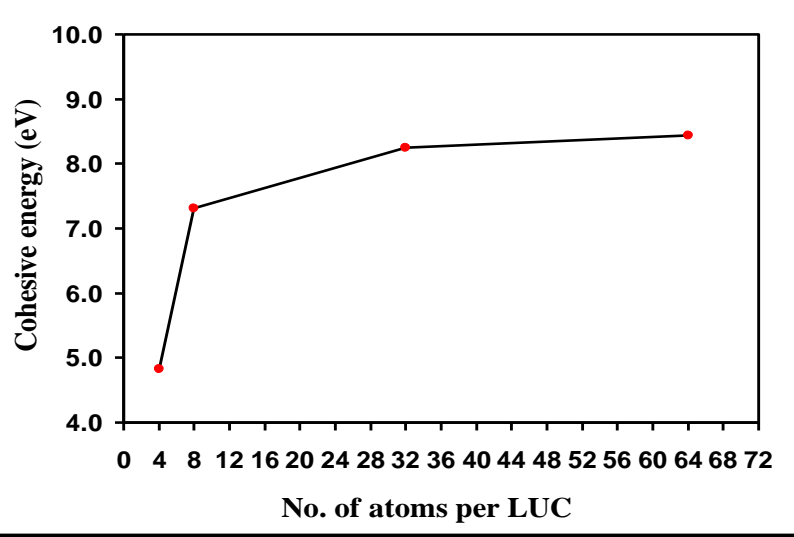

Figure 5:- the cohesive energy of In $\mathrm{N}$ nanocrystal as a function of number of core atoms. 
The electron affinity $\left(\chi_{e}\right)$ of InN nanocrystal lies between $3.199 \mathrm{eV}$ and $6.33 \mathrm{eV}$ and the ionization potential $\left(I_{P}\right)$ is between $3.109 \mathrm{eV}$ and $6.319 \mathrm{eV}$ for $4-64$ atoms. These values in good agreement with the results obtained by Jol w. ager et al. [37]. The electron affinity and the ionization potential as a function of number of core atoms is shown in Figs. (6) and (7), respectively.

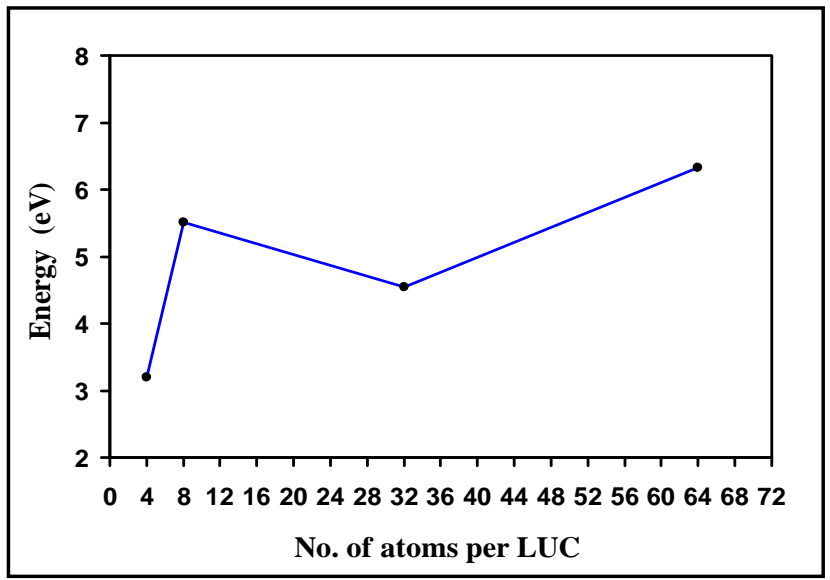

Figure 6:- shows the electron affinity as a function of the number of core atoms.

We can notice the fluctuation and disparate behavior of the electron affinity and the ionization potential with variation the core of atoms. This behavior returns to the shape effect of the structure [12,14], where the LUC of 4 and 32 atoms have hexagonal multiples structure while LUC of 8 and 64 atoms have orthorhombic multiples structure. This behavior is noted in many studies [38].

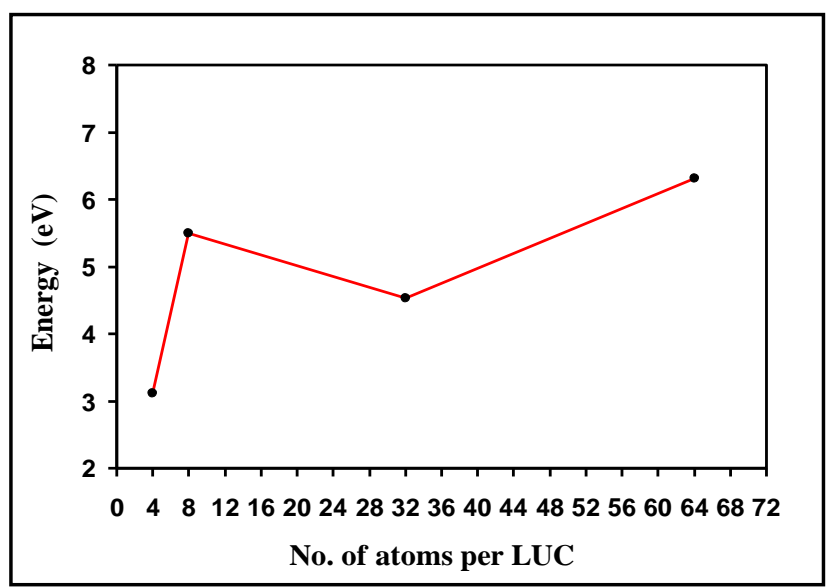

Figure 7:- the ionization potential as a function of the number of core atoms

Fig. (8) illustrate the density of states (DOS) as a function of orbital energy. The degeneracy of states has been maximum of 1 for 4 core atoms and 4 for 64 core atoms respectively for considered nanocrystals. Highly degenerate states seen in the core reflects high symmetry, equal bond lengths and angles in perfect structure. Finally, table (1) summarized the structural and electronic properties of InN wurtzite nanocrystal for many different LUCs. 

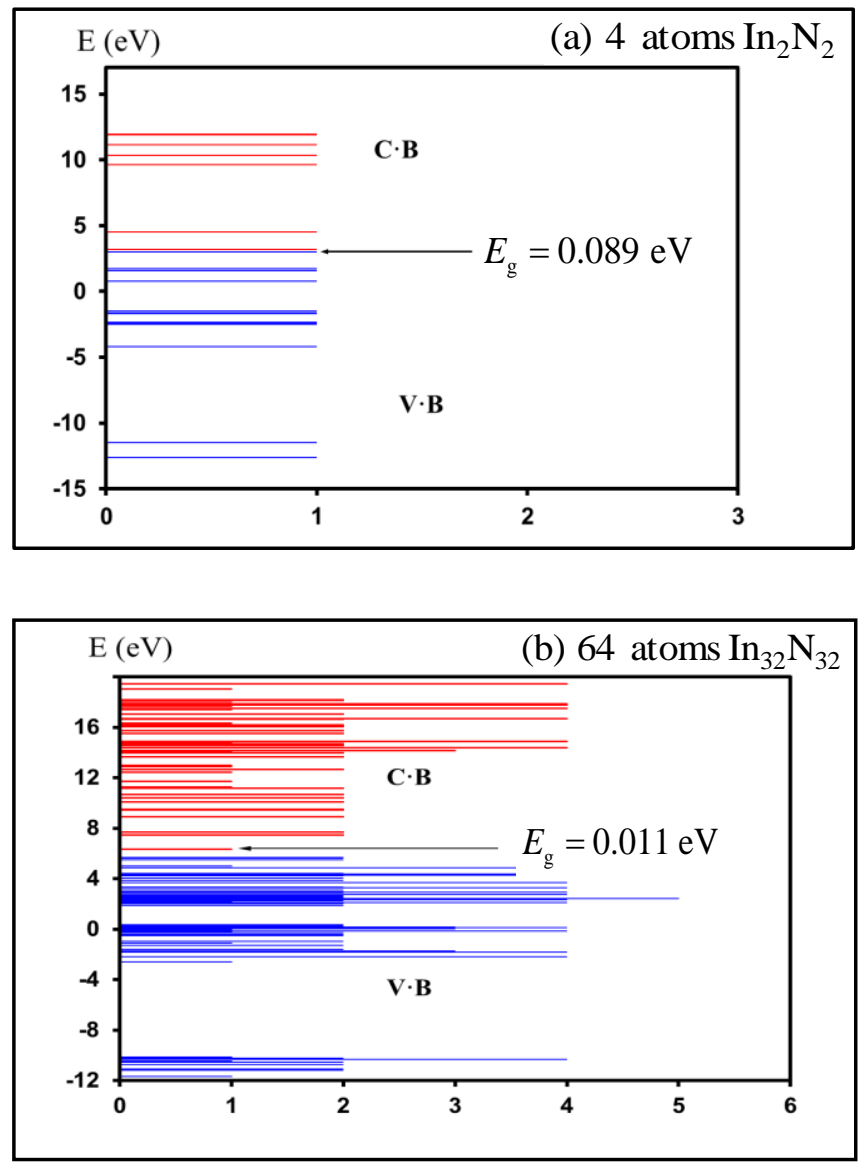

Figure 8:- Density of states of (a) 4 and (b) 64 atoms of core part of InN nanocrystals. Valence band is shown with blue lines while conduction band is shown with red lines. The energy gap is shown between the two bands.

Table 1:- structural and electronic properties of $\mathrm{InN}$ wurtzite nanocrystal.

\begin{tabular}{|c|c|c|c|c|c|c|}
\hline $\begin{array}{c}\text { Core atoms } \\
\text { of LUC }\end{array}$ & $\begin{array}{c}a \\
(\mathbf{n m})\end{array}$ & $\begin{array}{c}c \\
(\mathbf{n m})\end{array}$ & $\begin{array}{c}\boldsymbol{E}_{g} \\
(\mathbf{e V})\end{array}$ & $\begin{array}{c}\chi_{e} \\
(\mathrm{eV})\end{array}$ & $\begin{array}{c}I_{P} \\
(\mathrm{eV})\end{array}$ & $\begin{array}{c}\boldsymbol{E}_{\text {coh }} \\
(\mathrm{eV} / \text { atom })\end{array}$ \\
\hline 4 & $\mathbf{0 . 3 7 1}$ & $\mathbf{0 . 5 9 7 3 1}$ & $\mathbf{0 . 0 8 9}$ & $\mathbf{3 . 1 9 9}$ & $\mathbf{3 . 1 0 9}$ & $\mathbf{4 . 8 3 4}$ \\
\hline 8 & $\mathbf{0 . 3 4 7}$ & $\mathbf{0 . 5 5 8 6 7}$ & $\mathbf{0 . 0 0 7}$ & $\mathbf{5 . 5 0 8}$ & $\mathbf{5 . 5 0 1}$ & $\mathbf{7 . 3 1 7}$ \\
\hline 32 & $\mathbf{0 . 3 4 1}$ & $\mathbf{0 . 5 4 9 0 1}$ & $\mathbf{0 . 0 0 4}$ & $\mathbf{4 . 5 4 2}$ & $\mathbf{4 . 5 3 7}$ & $\mathbf{8 . 2 4 5}$ \\
\hline 64 & $\mathbf{0 . 3 4 0}$ & $\mathbf{0 . 5 4 7 4 0}$ & $\mathbf{0 . 0 1 1}$ & $\mathbf{6 . 3 3 0}$ & $\mathbf{6 . 3 1 9}$ & $\mathbf{8 . 4 3 9}$ \\
\hline
\end{tabular}

\section{Conclusion:-}

We can conclude that many of the physical properties will be changed when the size of particle become several nanometer because of the quantum confinement effect which can be observed when the size of the particle is too small to be comparable to the wavelength of the electron. All the studied properties are different from the bulk InN. The energy gap, lattice constant and energy gap are size dependent.

\section{Acknowledgements:-}

The researchers acknowledges the physics department-Koya University for their support to this work by using the computer lab. 


\section{References:-}

1. D. A. Neumayer and J.G. Ekerdt, Growth of Group III Nitrides. A Review of Precursors and Techniques, Chem. Mater. 8, 9-25 (1996)

2. W. Walukiewicz, J.W. Ager III, K.M. Yu, Z. Lilienthal-Weber, J. Wu, S. X. Li, R. E. Jones and J. D. Denlinger, Structure and electronic properties of InN and In-rich group III-nitride alloys, J. Phys. D: Appl. Phys 39 (2006)

3. I. Vurgaftman, J. R. Meyer, Band parameters for nitrogen-containing semiconductors, J. Appl. Phys. 94, 3675 (2003)

4. K. A. Mkhoyan, J. Silcox, E. S. Alldredge and N. W. Ashcroft, Measuring electronic structure of wurtzite InN using electron energy loss spectroscopy, Appl. Phys. Lett., Vol. 82, No. 9 (2003)

5. V. Yu. Davydov, V. V. Emtsev, I. N. Goncharuk, A. N. Smirnov, V. D. Petrikov, V. V. Mamutin, V. A. Vekshin, S. V. Ivanov, M. B. Smirnov, and T. Inushima, Experimental and theoretical studies of phonons in hexagonal InN , Appl. Phys. Lett. 75, 3297 (1999)

6. E. Kurimoto, H. Hamira, A. Hashimoto, and A. Yamamoto, MOCVD Growth of High-Quality InN Films and Raman Characterization of Residual Stress Effects, Phys. Status Solidi B 228, 1 (2001)

7. H. Lu, W. J. Schaff, J. Hwang, H. Wu, G. Koley, and L. F. Eastman, Effect of an AlN buffer layer on the epitaxial growth of InN by molecular-beam epitaxy, Appl. Phys. Lett. 79, 1489 (2001)

8. S. Strite and H. Morkoc, GaN, AIN, and InN: A review, J. Vac. Sci. Technol. B 10, 1237 (1992)

9. Akram H. Taha, Electronic Structure Simulation of Aluminum Antimony Nanocrystal Using Ab-initio Density Functional Theory coupled with Large Unit Cell Method, American Journal of Condensed Matter Physics 2014, 4(4): 63-70, (2014).

10. F. Annane, H. Meradji , S. Ghemid, F. El Haj Hassan, First principle investigation of AlAs and AlP compounds and ordered AlAs ${ }_{1-x} \mathbf{P}_{\mathbf{x}}$ alloys, Computational Materials Science 50 274-278, (2010).

11. M T Hussein, $\mathrm{T}$ Kasim and $\mathrm{M} A$ Abdulsattar First principle study of electronic nanoscale structure of $\mathbf{I n}_{\mathbf{x}} \mathbf{G a}_{1-\mathrm{x}} \mathbf{P}$ with variable size, shape and alloying percentage, Indian J. Phys. 87(11):1079-1085, (2013)

12. A H Harker and F P Larkins, A large unit cell semi-empirical molecular orbital approach to the properties of solids. II. Covalent materials: diamond and silicon, J. Phys. C: Solid State Phys. 12 2497-2508 (1979)

13. A. M. Dobrotvorskii and R. A. Evarestov, The Quasi-Molecular Large Unit Cell Model in the Theory of Deep Levels in Imperfect Crystals: Point Defects in Graphitic Boron Nitride, Phy. Stat. Solidi b 66 83-91, (1974),

14. A. H. Harker and F. P. Larkins, A large unit cell semi-empirical molecular orbital approach to the properties of solids. I. General theory. J. Phys. C: Solid State Phys., Vol. 12:2487-2495, (1979),

15. Frank Jensen, Introduction to Computational Chemistry, John Wiley \& Sons, Inc., 2nd edition (2007)

16. L. H. Thomas, The calculation of atomic fields. Proc. Cambridge Philos. Soc. 23: 542, (1927).

17. Swapan K. Ghosh, Density Functional Theory and Materials Modeling at Atomistic Length Scales, Int. J. Mol. Sci., 3, 260-275, (2002).

18. M. V. Putz and D. M. Mingos, Applications of Density Functional Theory to Biological and Bioinorganic Chemistry, Springer-Verlag Berlin Heidelberg, (2013).

19. P. Hohenberg and W. Kohn, Inhomogeneous Electron Gas, Physical Review, vol. 136, no. 3B (1964).

20. W. Kohn and L. J. Sham, Self-Consistent Equation Including Exchange and Correlation Effects, Reviews of Modern Physics, Vol. 140, No. 4A (1965).

21. Cle'mence Corminboeuf, Fabien Tran and Jacques Weber, The Role of Density Functional Theory in Chemistry: Some Historical Landmarks and Applications to Zeolites, Journal of Molecular Structure: THEOCHEM 762, 1-7 (2006)

22. John A. Pople, Peter M. W. and Benny G. Johnson, Kohn-Sham Density-Functional Theory within a Finite Basis Set, Chemical Physics Letters, Vol. 199, No. 6 (1992)

23. J. P. Perdew, K. Burke and M. Ernzerhof, Generalized gradient approximation made simple, Physical Review Letters, Vol. 77, No. 18 (1996)

24. John P. Perdew, AdriennRuzsinszky, Jianmin Tao, Viktor N. Staroverov, Gustavo E. Scuseria and Gábor I. Csonka, Prescription for the Design and Selection of Density Functional Approximations: More Constraint Satisfaction with Fewer Fits, Journal of Chemical Physics, 123, 062201 (2005)

25. M. J. Frisch, G.W. Trucks, H.B. Schlegel, G. E. Scuseria, M. A. Robb, J. R. Cheeseman, J. A. Montgomery Jr., T. Vreven, K. N. Kudin, J. C. Burant, J. M. Millam, S. S. Iyengar, J. Tomasi, V. Barone, B. Mennucci, M. Cossi, G. Scalmani, N. Rega, G.A. Petersson, H. Nakatsuji, M. Hada, M. Ehara, K. Toyota, R. Fukuda, J. Hasegawa, M. Ishida, T. Nakajima, Y. Honda, O. Kitao, H. Nakai, M. Klene, X. Li, J.E. Knox, H.P. Hratchian, J.B. Cross, C. Adamo, J. Jaramillo, R. Gomperts, R.E. Stratmann, O. Yazyev, A.J. Austin, R. Cammi, C. 
Pomelli, J. W. Ochterski, P.Y. Ayala, K. Morokuma, G.A. Voth, P. Salvador, J.J. Dannenberg, V. G. Zakrzewski, S. Dapprich, A. D. Daniels, M. C. Strain, O. Farkas, D. K. Malick, A. D. Rabuck, K. Raghavachari, J. B. Foresman, J. V. Ortiz, Q. Cui, A. G. Baboul, S. Clifford, J. Cioslowski, B. B. Stefanov, G. Liu, A. Liashenko, P. Piskorz, I. Komaromi, R. L. Martin, D. J. Fox, T. Keith, M.A. Al-Laham, C. Y. Peng, A. Nanayakkara, M. Challacombe, P. M. W. Gill, B. Johnson, W. Chen, M. W. Wong, C. Gonzalez, J. A. Pople, Gaussian 03w, Revision B.01, Gaussian, Inc., Pittsburgh PA (2003).

26. A. P. Alivisatos, Perspectives on the Physical Chemistry of Semiconductor Nanocrystals, J. Phys. Chem. 100, 13226-13239 (1996).

27. Z. Hens, D. Vanmaekelbergh, E. J. A. J. Stoffels, H. van Kempen, Effects of Crystal Shape on the Energy Levels of Zero-Dimensional PbS Quantum Dots, Phys. Rev. Lett. 88, 236803, (2002).

28. K. Sattler, Nanomaterials and Magnetic Thin Films, vol. 5, Academic Press, New York, p. 61, (2002).

29. R. Erni and Nigel D. Browning, Quantification of the size-dependent energy gap of individual CdSe quantum dots by valence electron energy-loss spectroscopy, Ultramicroscopy 107 267-273, (2007).

30. F. Bechstedt, J. FurthmKller, Do we know the fundamental energy gap of InN? J. Cryst. Growth, 246,315 319, (2002).

31. J. Wu, W. Walukiewicz, K. M. Yu, J.W. Ager III, E. E. Haller, H. Lu, W. J.Schaff, Y. Saito, Y. Nanishi, Unusual properties of the fundamental band gap of InN, Appl. Phys. Lett.,80,3967-3969, (2002).

32. Kripasindhu Sardar,F. L. Deepak, A.Govindaraj, M. M. Seikh, and C. N. R. Rao, InN Nanocrystals, Nanowires, and Nanotubes, small , 1, No. 1, $91-94$, (2005).

33. A. S. Verma, B. K. Sarkar and V. K. Jindal, Cohesive energy of zincblende $\left(A^{\mathrm{III}} \mathbf{B}^{\mathrm{V}}\right.$ and $\left.A^{\mathrm{II}} \mathbf{B}^{\mathrm{VI}}\right)$ structured solids. Pramana - J. Phys., Vol. 74, No. 5; pp. 851-855, (2010).

34. Askander K. Kaka, "Size Variation Effect of $\operatorname{In}_{1-x} \mathbf{A l}_{\mathbf{x}} \mathbf{P}$ Nanocrystal Alloying Composition Using Density Functional Theory", University of Baghdad, Ph.D Thesis, (2015).

35. M. Fuchs, J. L. F. Da Silva, C. Stampfl, J. Neugebauer, and M. Scheffler, Cohesive properties of group-III nitrides: A comparative study of all-electron and pseudopotential calculations using the generalized gradient approximation, Phys. Rev. B, Vol. 65, 245212, (2002).

36. A. F. Wright and J.S. Nelson, Consistent structural properties for AIN, GaN, and InN, Phys. Rev. B 51, 7866 (1995);50, 2159 (1994)

37. Joel W. Ager, nate R. Miller, Taming transport in InN, Physica Status solidi, Vol. (a) 209, PP 83-86, (2012).

38. Mudar A. Abdulsattar, Mesoscopic Fluctuations of Electronic Structure Properties of Boron Phosphide Nanocrystals. Electronic Materials Letters, Vol. 6, No. 3, pp. 97-101 (2010). 